Article abstract-One method of evaluating the degree of neurologic impairment in MS has been the combination of grades $(0=$ normal to 5 or $6=$ maximal impairment) within 8 Functional Systems (FS) and an overall Disability Status Scale (DSS) that had steps from 0 (normal) to 10 (death due to MS). A new Expanded Disability Status Scale (EDSS) is presented, with each of the former steps $(1,2,3 \ldots 9)$ now divided into two $(1.0,1.5,2.0 \ldots 9.5)$. The lower portion is obligatorily defined by Functional System grades. The FS are Pyramidal, Cerebellar, Brain Stem, Sensory, Bowel \& Bladder, Visual, Cerebral, and Other; the Sensory and Bowel \& Bladder Systems have been revised. Patterns of FS and relations of FS by type and grade to the DSS are demonstrated.

NEUROLOGY (Cleveland) 1983;33:1444-52

\title{
Rating neurologic impairment in multiple sclerosis: An expanded disability status scale (EDSS)
}

\author{
John F. Kurtzke, MD
}

In 1955 I described "a new scale for evaluating disability in multiple sclerosis," later known as the Disability Status Scale (DSS), devised to evaluate isoniazid as a possible treatment. ${ }^{2}$ This scale was also used in the first multicentered, randomized, placebocontrolled, double-blind trial of MS therapy, ${ }^{3}$ which refuted our original claim, a decision with which we had to concur from our later experience. ${ }^{4}$ The DSS had 10 grades or steps beyond 0 (normal), extending to status 10 (death due to MS). The scale was "intended to measure the maximal function of each patient as limited by ... neurologic deficits,"1 and it was based on neurologic examination.

The DSS was later made half of a bifid rating system, the other part "being a series of grades in each of eight functional groupings.... In each portion, there is a numerical rating which is mutually exclusive in its category, and the higher the number, the greater is the dysfunction. Only objectively verifiable defects due to multiple sclerosis as elicited upon neurologic examination are included. Symptoms are discarded." ".

The functional groups, later called Functional Systems (FS), were Pyramidal (P), Cerebellar (Cll), Brain Stem (BS), Sensory (S), Bowel \& Bladder (BB), Visual (V), Cerebral or Mental (Cb), and Other or Miscellaneous (O) Functions. All save the last were graded from 0 (normal) to maximal impairment (grade 5 or 6 ); the "Other" FS was dichotomous, with 0 as none and 1 as any present. Approximate equivalents for the DSS steps were also provided. The Functional Systems were mutually exclusive in terms of neuroanatomy, but together comprised all neurologic abnormalities on examination that can be attributed to MS lesions. The FS were not additive; each FS could be compared over time only with itself, and for this reason it was necessary to retain the DSS for overall comparisons of the same patient at different examinations.

The FS were modified in 1965 by changing the Sensory scale from $0-5$ to $0-6$ and redefining the upper grades for Bowel \& Bladder. ${ }^{6}$ As will be seen below, the Sensory System is again being revised, and Bowel \& Bladder has a new step.

This two-part system of assessing neurologic impairment in MS has been used in several studies, and it has been proposed for adoption as one part of a tridimensional scheme for a "minimal data set" in MS, which will be discussed below. However, some investigators believe the DSS is too insensitive to change in the middle ranges, and have urged division of step 7 into two parts. Further, while the DSS was considered satisfactory in several treatment trials in acute bouts, it was thought that there should be more

From the Neurology Service, Veterans Administration Medical Center and Departments of Neurology and of Community Medicine, Georgetown University School of Medicine, Washington, DC.

Accepted for publication February 9, 1983.

Address correspondence and reprint requests to Dr. Kurtzke, Chief, Neurology Service (127), Veterans Administration Medical Center, 50 Irving Street, NW, Washington, DC 20422 
room for change in studies of chronic MS.

For these reasons, an Expanded DSS (EDSS) is now presented. It provides, for each step from 1 through 9, two steps that together add up to the same step of the original DSS. This division relies even more heavily on the standard neurologic examination as encoded in the Functional Systems. In fact, it is fully defined in the lower ranges by the FS grades. For this reason, before presenting the Expanded DSS, we need to consider the Functional Systems.

Functional Systems. The grades for each of the Functional Systems are defined in appendix A. They are identical with those provided in $1965^{6}$ except for the new Sensory and Bowel \& Bladder Systems. The frequency of involvement in each system at admission to the hospital for an early bout of MS in one series is described in table $1 .^{7}$

Recall that each FS is independent of the others, yet together they reflect all neurologic impairment in MS. There are over 1.3 million possible patterns of involvement by FS type and grade. However, if we consider each System as just involved (1) or not involved (0), then neurologic impairment can be defined by an eight-digit binary number. For example, a patient with Pyramidal, Cerebellar, and Sensory signs, the other Systems normal, would be described as 11010000 . There are then only 256 possible patterns $\left(2^{8}\right)$ into which a patient can fall. From the same series as in table 1 , there are described the most common patterns to be expected if lesions in one system were independent of lesions in the others (table 2). These expected frequencies compare well with those actually observed for the same specific patterns. ${ }^{8}$ One-half of the patients fell into one of only 14 patterns, and $1 / 4$ into one of only 4 patterns.

Several points of clarification may be in order for the Functional Systems. Pyramidal, Cerebellar, Sensory, and Bowel \& Bladder functions all refer to impairment of body parts below the head only (regardless of the site of the lesions), and Brain Stem functions have always referred to impairment "attributable to lesions of supra- and intersegmental tracts subserving cranial nerves 3 through 12 , together with involvement of these nuclei or their intramedullary fibers. These, therefore ... encompass pseudobulbar palsies and scanning speech ... in addition to the so-called cranial nerve functions."

For each FS and the DSS, the rule remains: "Where criteria for the precise grade are not met, the nearest appropriate category is utilized." ${ }_{5}$ Thus Pyramidal grade 5 would be used rather than 4 for one who is almost paraplegic. Whatever the specific grade definition, then, "almost" or "practically" can be prefixed. One method for difficult decisions is to "bracket" the likely grade and then cone down on the most applicable.

The Expanded Disability Status Scale. The EDSS (appendix B) will be discussed under con-
Table 1. Percentage frequency of involvement according to Functional Systems (FS) from neurologic examinations at admission to hospital for an early bout of MS; Army WW II series*

\begin{tabular}{|c|c|c|}
\hline $\begin{array}{l}\text { Functional } \\
\text { Systems (FS) }\end{array}$ & \% involved & $\begin{array}{c}\text { Total } \\
\text { N known }\end{array}$ \\
\hline Pyramidal (P) & 84.9 & 511 \\
\hline Cerebellar (CII) & 76.9 & 481 \\
\hline Brain Stem (BS) & 73.0 & 514 \\
\hline Sensory (S) & 55.2 & 478 \\
\hline Bowel \& Bladder (BB) & 22.6 & 517 \\
\hline $\mathrm{Visual}^{+}(\mathrm{V})$ & 33.9 & 425 \\
\hline Cerebral-total ${ }^{(\mathrm{Cb})}$ & 20.7 & 487 \\
\hline Cerebral-mentation ${ }^{\S}$ & 2.9 & 487 \\
\hline Other $(\mathrm{O})$ & 14.9 & 523 \\
\hline \multicolumn{3}{|c|}{$\begin{array}{l}\text { * From Kurtzke et al, Acta Neurol Scand 1972;48:19-46. } \\
\text { + Neuropathic signs either/both eyes; see *. } \\
\text { † Includes mood changes only (step 1). } \\
\text { \$ Steps } 2+\text { on the scale. }\end{array}$} \\
\hline
\end{tabular}

Table 2. Patterns of involvement by Functional System (FS) from neurologic examinations at admission to hospital for an early bout of MS; Army WWII series*

\begin{tabular}{|c|c|c|c|c|c|}
\hline \multirow[b]{2}{*}{ Rank $^{\dagger}$} & \multirow[b]{2}{*}{ Pattern ${ }^{\ddagger}$} & \multicolumn{2}{|c|}{ No. of cases } & \multicolumn{2}{|c|}{ Cumulative $p^{\S}$} \\
\hline & & 0 & $\mathbf{E}$ & 0 & $\mathbf{E}$ \\
\hline 1 & 11110000 & 31 & 28.92 & 0.093 & 0.086 \\
\hline 2 & 11100000 & 29 & 28.74 & 0.179 & 0.172 \\
\hline 3 & 11110100 & 12 & 15.32 & 0.215 & 0.218 \\
\hline 4 & 11100100 & 16 & 15.23 & 0.263 & 0.263 \\
\hline 5 & 11010000 & 14 & 9.37 & 0.304 & 0.291 \\
\hline 6 & 11000000 & 8 & 9.32 & 0.328 & 0.319 \\
\hline 7 & 10110000 & 6 & 8.78 & 0.346 & 0.345 \\
\hline 8 & 10100000 & 7 & 8.72 & 0.367 & 0.371 \\
\hline 9 & 11110010 & 15 & 7.78 & 0.412 & 0.395 \\
\hline 10 & 11100010 & 8 & 7.73 & 0.436 & 0.418 \\
\hline 11 & 11111000 & 11 & 7.09 & 0.469 & 0.439 \\
\hline 12 & .11101000 & 4 & 7.05 & 0.481 & 0.460 \\
\hline 13 & 01110000 & 1 & 6.18 & 0.484 & 0.478 \\
\hline 14 & 01100000 & 9 & 6.15 & 0.510 & 0.497 \\
\hline $15-256$ & all other & 164 & 168.63 & 1.000 & 1.000 \\
\hline 256 & Total & 335 & 335.01 & 1.000 & 1.000 \\
\hline \multicolumn{6}{|c|}{$\begin{array}{l}\text { * Adapted from Kurtzke, Acta Neurol Scand 1970;46:493-512. } \\
\text { Rank order of expected frequency of specific pattern, based upon } \\
\text { product of individual observed frequencies with hypothesis of } \\
\text { independence for all patterns where } \mathrm{E} \geq 5.0 ; \mathrm{O}=\text { observed and } \\
\mathrm{E}=\text { expected frequency. } \chi^{2}, 20,58, p>0.10 \text { for } \mathrm{O} \text { versus } \mathrm{E} \text {. } \\
\text { Involved }(1) \text { or not involved }(0) \text { for } \mathrm{P}, \mathrm{Cll}, \mathrm{BS}, \mathrm{S}, \mathrm{BB}, \mathrm{V}, \mathrm{Cb}, \mathrm{O} \text { in } \\
\text { cited order; cases with complete information on all } 8 \mathrm{FS} \text {. } \\
\text { Cumulative proportion }(p) \text { of total, observed }(\mathrm{O}) \text {, and expected } \\
\text { (E) patterns. }\end{array}$} \\
\hline
\end{tabular}


Table 3. Percentage frequency distribution of Functional System (FS) grades according to DSS steps. I: DSS 1-2*

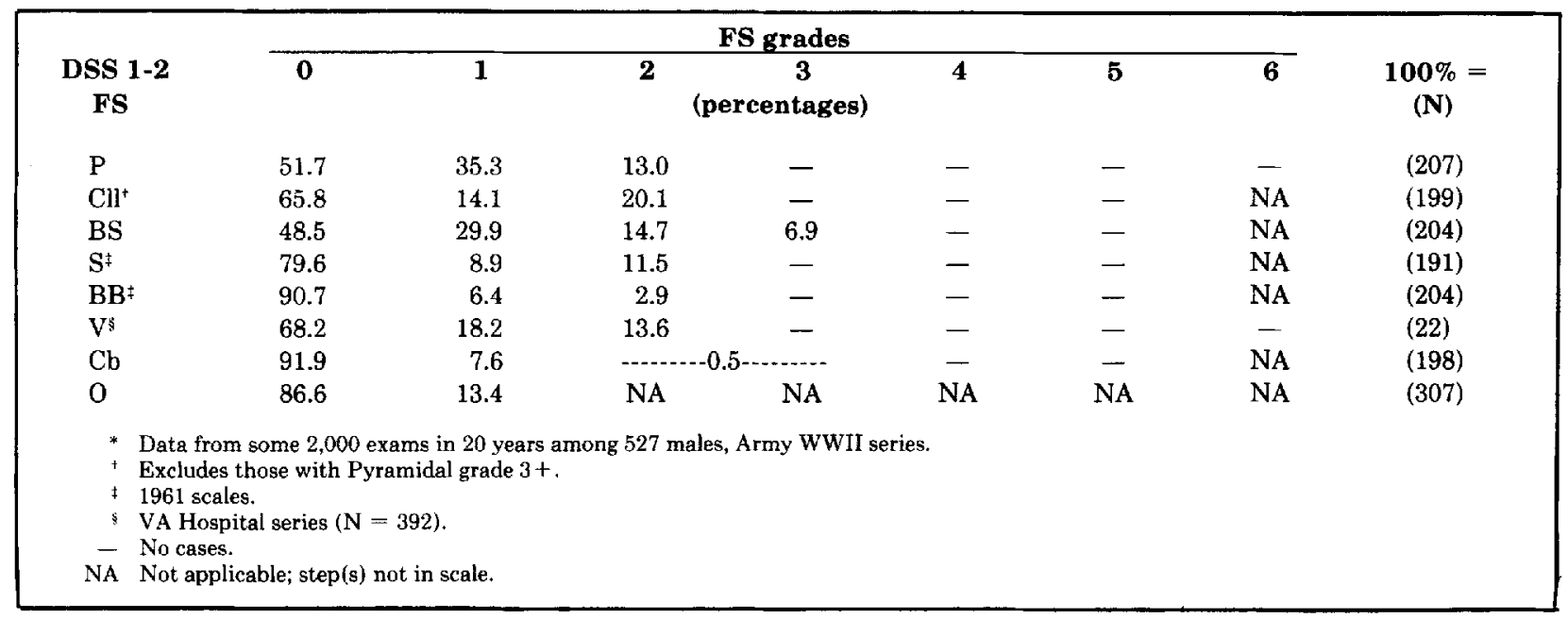

secutive groupings of the original DSS. For this expansion, we have had to make more finite and arbitrary distinctions than in the original scale.

DSS Step 0. As before, this defines the normal neurologic examination-regardless of symptoms. Therefore, all FS are grade 0, except for Cerebral System grade 1. Cerebral "grade 1 refers to mood aberrations such as euphoria or depression, which may not be a primary effect of the disease process, but this is hoped to represent that stage of brain damage when alterations of personality or emotional control are the sole features." For DSS step 0 and step 1, Cerebral grade 1 is treated as a 0 .

DSS Steps 1-2. These steps refer to minimal objective abnormality, with step 1 as signs without impaired function. Table 3 shows the distribution of FS grades for DSS 1-2 from an overview of some 20 years' follow-up examinations in 527 men with MS, our Army WW II series. ${ }^{8}$ The ratio of step 2 to 1 was about 2:1. The DSS scores in this series were not strictly delimited by the FS equivalents described here. Nevertheless, the low frequency of involvement is evident; this was essentially limited to FS grades 1 and 2 except for the $7 \%$ in Brain Stem grade 3 . The FS scales used here and below are the 1961 variants for Sensory and Bowel \& Bladder.

EDSS Step 1.0 is limited to one FS grade 1, excluding Cerebral grade 1, with all others grade 0.

EDSS Step 1.5 is defined as two or more FS grade 1, again excluding Cerebral grade 1, but no grade above 1 in any FS.

EDSS Step 2.0 is limited to one FS grade 2, others grade 0 or 1 .

EDSS Step 2.5 is limited to two FS grade 2, others grade 0 or 1 .

Note that it is irrelevant which FS are involved, and from table 3 , it is likely to be any of them except Bowel \& Bladder or Cerebral.

DSS Steps 3-4. These steps still refer to mild disorder, not sufficient to impede normal activities of daily living or work in most situations. However, a concert pianist, a pilot, or a steeplejack would doubtless not be able to function as usual and still be ascribable to these steps. Full ambulation-meaning ability to be up and about all day and to walk usual distances without resting-characterize these steps. Impaired ambulation of any degree should not occur with FS grades defining DSS step 3 . There is some overlap of FS in steps 4 and 5 . Table 4 delineates the distribution of FS grades for DSS 3-4. The ratio of step 3 to 4 was about unity. Only rarely was grade 4 attained. We begin to see the predominance of Pyramidal involvement, closely followed by Cerebellar and Brain Stem.

EDSS Step 3.0 is limited to one FS grade 3, or three or four FS 2, others being 0 or 1 .

EDSS Step 3.5 is limited to one FS grade 3 plus one or two grade 2, or two FS grade 3, or five FS grade 2 , others being grade 0 or 1 .

EDSS Step 4.0 consists of combinations just exceeding two grade 3 , or one grade 3 plus two grade 2 , or five grade 2; or one FS grade 4 alone, all others being grade 0 or 1 . At this point, the ambulation/ work/daily activity abilities start to take precedence over the precise FS grades. With FS that exceed the criteria for EDSS step 3.5, there must be, for step 4.0, full ambulation (including ability to walk without aid or rest for some 500 meters), and ability to carry out full daily activities to include work of average physical difficulty.

EDSS Step 4.5 has the same minimal FS grade requirements as step 4.0. The patient must be able to walk without aid or rest for some 300 meters and to work a full day in a position of average difficulty. The patient is up and about most of the day, but some limitation of full activity separates this from step 4.0.

DSS Steps 5-6. The patient is not ordinarily housebound and can walk. Seldom is a full work day possible without special provisions. The original DSS 5 was defined as "maximal motor function 
Table 4. Percentage frequency distribution of Functional System (FS) grades according to DSS steps. II: DSS 3-4*

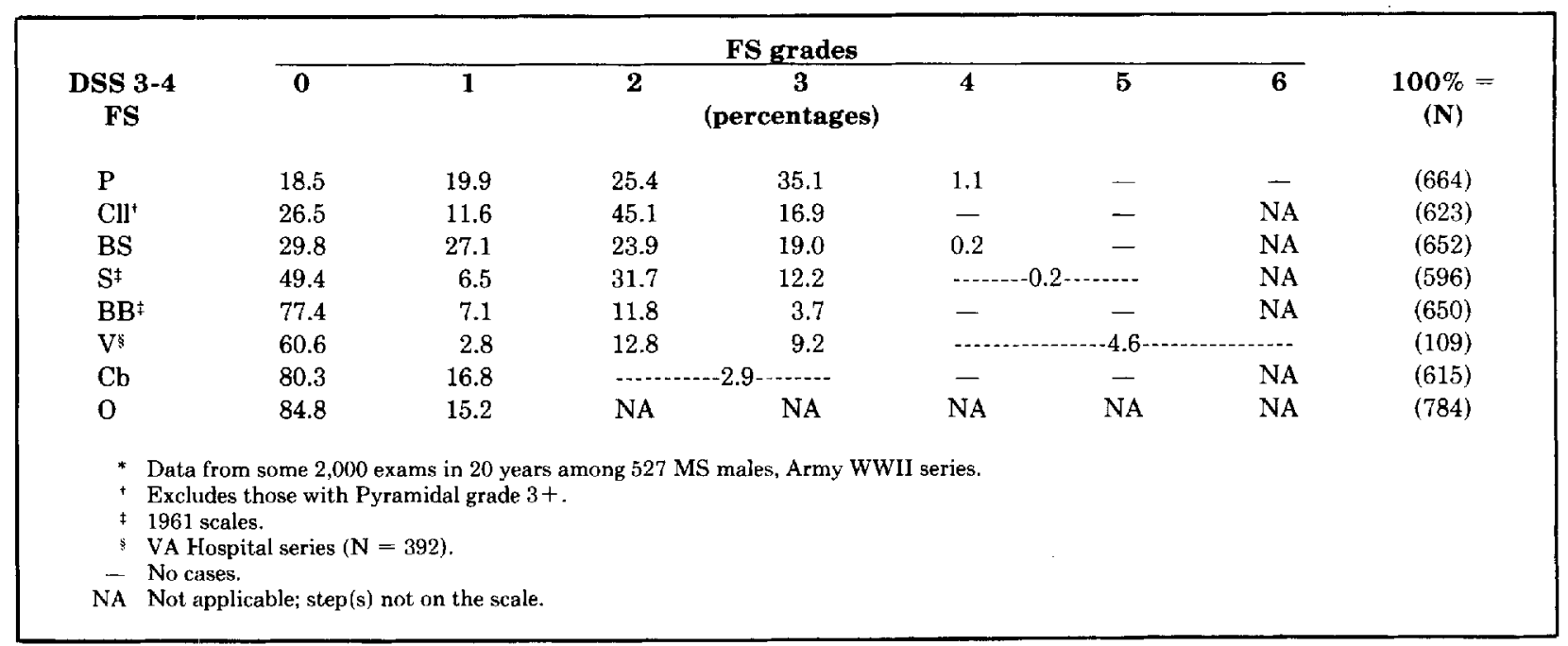

Table 5. Percentage frequency distribution of Functional System (FS) grades according to DSS steps. III: DSS 5-6*

\begin{tabular}{|c|c|c|c|c|c|c|c|c|}
\hline \multirow[b]{2}{*}{$\begin{array}{c}\text { DSS 5-6 } \\
\text { FS }\end{array}$} & \multicolumn{7}{|c|}{ FS grades } & \multirow[b]{2}{*}{$\begin{array}{r}100 \%= \\
(\mathrm{N})\end{array}$} \\
\hline & 0 & 1 & 2 & $\begin{array}{c}3 \\
\text { (percentages) }\end{array}$ & 4 & 5 & 6 & \\
\hline $\mathrm{P}$ & 2.1 & 6.6 & 8.5 & 49.5 & 32.8 & 0.5 & - & $(424)$ \\
\hline $\mathrm{Cll}^{+}$ & 5.6 & 2.5 & 24.9 & 56.7 & 10.3 & - & NA & $(358)$ \\
\hline BS & 19.2 & 23.1 & 30.2 & 26.0 & 1.5 & - & NA & $(407)$ \\
\hline $\mathrm{S}^{\ddagger}$ & 29.8 & 7.2 & 40.3 & 22.4 & $-\cdots-$ & -.... & NA & $(362)$ \\
\hline $\mathrm{BB}^{\ddagger}$ & 59.3 & 10.5 & 17.1 & 10.1 & 1.0 & 2.0 & NA & (398) \\
\hline $\mathrm{Vs}$ & 60.8 & 6.4 & 15.2 & 11.2 & $\cdots$ & $-6.4-$ & …- & (125) \\
\hline $\mathrm{Cb}$ & 72.6 & 20.7 & $\ldots$ & -5.0 & 0.8 & 0.8 & NA & $(362)$ \\
\hline 0 & 72.1 & 27.9 & $\mathrm{NA}$ & $\mathrm{NA}$ & NA & NA & $\mathrm{NA}$ & (459) \\
\hline \multicolumn{9}{|c|}{$\begin{array}{l}\text { Data from some } 2,000 \text { exams in } 20 \text { years among } 527 \text { MS males, Army WWII series. } \\
+ \text { Excludes those with Pyramidal grade } 3+. \\
\neq 1961 \text { scales. } \\
\text { VA Hospital series. }\end{array}$} \\
\hline
\end{tabular}

walking unaided up to several blocks," and for 6 it was "assistance required for walking." There is generally some impairment in usual daily activities. Table 5 indicates for these steps the increasing frequency and severity of FS involvement, particularly Pyramidal and Cerebellar systems, with Brain Stem and Sensory not far behind. The ratio of step 5 to 6 was about 1.7:1. The principal discrimination among these four new EDSS steps rests with walking; the patient's statements about walking are ordinarily acceptable, but direct observation-and on more than one occasion-may be required. We are after "usual best function" here, and neither supramaximal nor insufficient efforts at performance. The FS equivalents are advisory and not prescriptive for these and higher steps.

EDSS Step 5.0 requires ambulation for about 200 meters without aid or rest. Disability is severe enough to impair full daily activities, eg, to work a full day without special provisions. Usual FS equivalents are one grade 5 alone, others 0 or 1 , or combinations of lesser grades that will usually exceed those specified for EDSS step 4.0.

EDSS Step 5.5 requires ambulation for some 100 meters without aid or rest. Other criteria are inability to work part-time (about $1 / 2$ day) without special provisions. Usual FS equivalents are as in step 5.0. Note the arbitrary distances for walking ability.

EDSS Step 6.0 requires assistance to walk about 100 meters. This may mean resting, the use of unilateral aids (cane, crutch, or brace) at most times, or the intermittent use of bilateral aids. The assistance of another person also counts as "with aid." The 
Table 6. Percentage frequency distribution of Functional System (FS) grades according to DSS steps. IV: DSS 7-9*

\begin{tabular}{|c|c|c|c|c|c|c|c|c|c|}
\hline \multirow{2}{*}{\multicolumn{2}{|c|}{$\begin{array}{c}\text { DSS } 7-9 \\
\text { FS }\end{array}$}} & \multicolumn{7}{|c|}{ FS grades } & \multirow[b]{2}{*}{$\begin{array}{r}100 \%= \\
(\mathrm{N})\end{array}$} \\
\hline & & 0 & $\mathbf{1}$ & $\mathbf{2}$ & $\begin{array}{c}3 \\
\text { (percentages) }\end{array}$ & 4 & 5 & 6 & \\
\hline $\mathbf{P}$ & & 0.7 & 1.3 & 1.0 & 3.0 & 40.6 & 45.9 & 7.5 & $(305)$ \\
\hline $\mathrm{Cll}^{+}$ & & 1.0 & - & 4.0 & 23.2 & 49.5 & 22.2 & NA & (99) \\
\hline BS & & 17.9 & 12.1 & 16.8 & 32.8 & 15.7 & 4.6 & $\mathrm{NA}$ & $(280)$ \\
\hline $\mathrm{S}^{\ddagger}$ & & 28.1 & 4.6 & 33.6 & 29.9 & $-\cdots$ & n...... & $\mathrm{NA}$ & $(217)$ \\
\hline $\mathrm{BB}^{\ddagger}$ & & 20.3 & 3.6 & 12.7 & 27.9 & 7.6 & 27.9 & NA & $(276)$ \\
\hline $\mathrm{V}^{\mathrm{s}}$ & & 54.1 & 4.4 & 8.1 & 17.0 & $\cdots$ & $-16.3-$ & --- & (135) \\
\hline $\mathrm{Cb}$ & & 67.9 & 17.4 & $\ldots$ & $-5.8---$ & 4.7 & 4.7 & $\mathrm{NA}$ & $(172)$ \\
\hline 0 & & 57.9 & 42.1 & NA & NA & NA & NA & NA & $(328)$ \\
\hline \multicolumn{10}{|c|}{$\begin{array}{l}\text { * Data from some } 2,000 \text { exams in } 20 \text { years among } 527 \mathrm{MS} \text { males, Army WWII series. } \\
\text { Excludes those with Pyramidal grade } 3+. \\
1961 \text { scales. } \\
\text { VA Hospital series }(\mathrm{N}=392) \\
\text { NA No cases. } \\
\text { NA } \\
\text { Not applicable; step(s) not on the scale. }\end{array}$} \\
\hline
\end{tabular}

primary measure for this step is the ability to walk with help for about 100 meters. Usual FS equivalents are combinations with more than two FS grade $3+$.

EDSS Step 6.5 requires assistance to walk about 20 meters without resting by means of aids (canes, crutches, braces, or people), which are generally bilateral and generally constantly necessary. Usual FS equivalents are as in 6.0 -combinations with more than two FS grade $3+$. A person who cannot walk 20 meters is functionally almost nonambulatory and should be considered close to DSS 7 .

DSS Steps 7-9. These are the severely involved patients who are almost invariably limited to wheelchair or bed. Table 6 demonstrates the marked shift to the right for FS grade involvement, particularly those functions having to do with ambulation. This behavior of groups of MS patients lends validity to a scoring system that stresses ambulation in the higher ranges; only in the most severe will the loss of upper limb and head functions be added. The ratio of the steps here was about 1.4:1:1.

The original definition of DSS step 7 was "restricted to wheelchair (able to wheel self and enter and leave chair alone).... It does not include the patient who is tied in the chair and perambulated." Conversely, ability to walk short distances is not sufficient to qualify for step 6 . The arbitrary limit for "short distances" is taken here as about 5 meters. This provides some leeway between EDSS step 6.5 (20 meters) and 7.0 (5 meters). As with the other grades, assignment is to that closest to his performance.

EDSS Step 7.0 defines essential restriction to wheelchair with inability to walk beyond about 5 meters even with aid. Patients can transfer alone (with mechanical aids if needed) and wheel the standard wheelchair; are able to be up and about in the chair some 12 hours a day; with the chair, are not housebound and may even be employed. Usual FS equivalents are combinations with more than one FS grade 4+; rarely, Pyramidal grade 5 alone.

EDSS Step 7.5 describes inability to take more than a few steps and, essentially, restriction to wheelchair. With or without aid, these patients can transfer. They can wheel themselves, but cannot carry on in standard wheelchair a full day. They may require motorized wheelchair for ability to be up and about in the chair. Usual equivalents are combinations with more than one grade $4+$.

EDSS Step 8.0. The original DSS 8 definition was "restricted to bed but with effective use of the arms ...; he can usually feed himself and perform part of his toilet."' In our setting, it has been standard procedure to get bed patients into chairs as much as possible, so that the horizontal posture was not a requirement for "bed patient." This (to me) obvious point has led to some confusion as to requirements for DSS 8.

EDSS Step 8.0 is defined as bed patients who may be in chair or (passively) in wheelchair for much of the day, and it is so specified in appendix B. Primarily, though, they retain many self-care functions and generally have effective use of the arms. Usual FS equivalents are combinations, generally grade $4+$ in several systems.

EDSS Step 8.5 are the bed patients who in daytime generally cannot tolerate prolonged periods in chair and are more often in bed, unless tied in the chair. Primarily, they still have some effective use of one or both arms and can perform some self-care functions, but less than for step 8.0. Usual FS equivalents are as in step 8.0.

EDSS Step 9.0 are the "helpless bed patients" who, however, can communicate and eat. They cannot perform self-care functions (such as feeding). Usual FS 
Table 7. DSS: Percentage frequency distribution in two series of MS patients at examination

\begin{tabular}{|ccc|}
\hline DSS & Series 1 $^{*}$ & Series 2 $^{\dagger}$ \\
& & \\
0 & 0.3 & 5.4 \\
1 & 0.6 & 3.7 \\
2 & 6.9 & 8.3 \\
3 & 12.6 & 19.8 \\
4 & 16.0 & 19.8 \\
5 & 16.0 & 15.7 \\
6 & 13.1 & 9.1 \\
7 & 18.3 & 7.7 \\
8 & 12.3 & 4.8 \\
9 & 4.0 & 5.6 \\
10 & NA & NA \\
& & 99.9 \\
Total & 100.1 & $(1,665)$ \\
N & $(350)$ & \\
* VA Hospital series, admission status (data are percentages). \\
'Army WWII series, overview for some 20 years (data are percentages). \\
\hline
\end{tabular}

equivalents are combinations, mostly grade $4+$.

EDSS Step 9.5 defines the totally helpless bed patients who cannot communicate effectively, eat, or swallow. Usual FS equivalents are combinations, almost all grade $4+$.

EDSS Step 10 is death due to MS. This may be an acute death due to "brainstem" involvement or to respiratory failure ${ }^{9}$ or death consequent to the chronic bedridden state with terminal pneumonia, sepsis, uremia, cardiorespiratory failure. It excludes intercurrent causes of death. Antemortem, the patient will ordinarily be DSS 9, sometimes 8 .

Discussion. The expanded DSS should answer the needs of those who felt constrained by too few steps in the original scale. The reason each step had to be divided, rather than only a few steps, may be seen in table 7, which shows the distribution of DSS scores in two series of MS patients. In our hands, at least, the distribution was reasonably Gaussian, and no single step stood out as markedly discrepant. With this evidence, the DSS could in fact be treated as a true numerical scale, with means and standard deviations, rather than the ordinal (rank) scale that is its basic structure. This would imply that DSS 6 is twice as "bad" as DSS 3.

In several studies, a clearly bimodal distribution of DSS scores was found. Comparing the individual FS scores with DSS in many of these (published and unpublished) suggests that the DSS scores below 6 had been assigned with little regard to FS grades. This should be obviated if the new EDSS is used-or even if the old DSS were retained, but with the FS equivalents given here. The sum of the two EDSS steps of the same number, eg, $2.0+2.5$, would be identical with the old DSS number, ie 2. In one unpublished study, one DSS step was dramatically higher than all others. I
MS: COURSE IN HOSPITAL - TOTAL SERIES

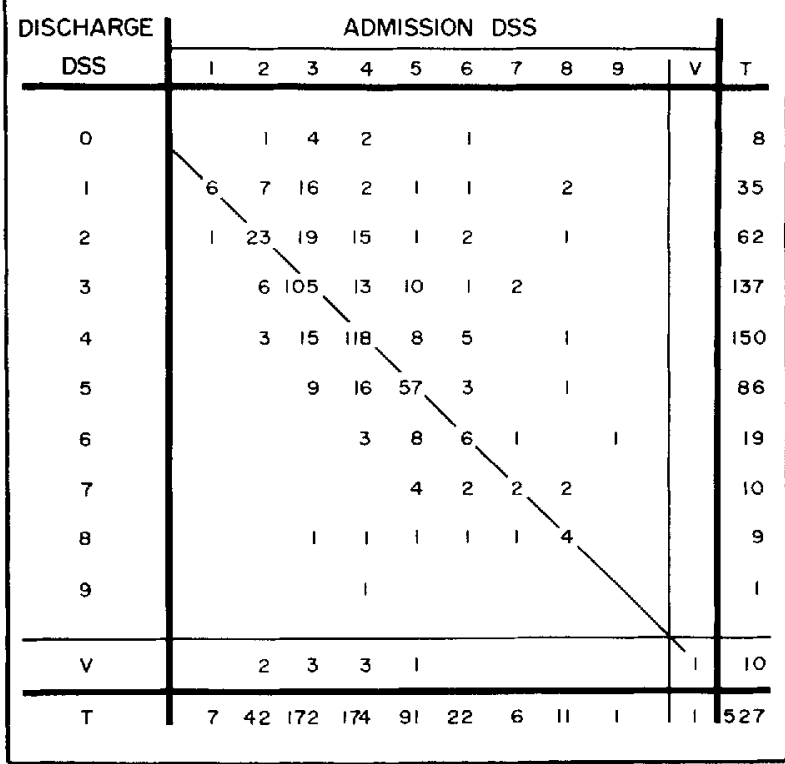

Figure. Grid correlate of DSS at admission to and discharge from hospital for an early bout of $M S$; Army $W W I I$ series. $^{7}$ Numbers along the main diagonal $(0,0$

9,9) indicate no change in DSS between admission and discharge; those above the diagonal improved and those below worsened, by the number of steps off diagonal for each locus.

suspect this was miscoding of contiguous steps, since nowhere else have I seen this.

The thesis that the DSS is a true numerical, equalinterval scale, though, is irrelevant to what I believe to be the proper handling of the scale as an index of neurologic change with time. To me, the Gaussian appearance is important principally in deciding that no one step is superfluous, and that no one step is really two or more steps on the continuum from normal to maximal disease. This appearance of a normal distribution is the basic reason for the EDSS as presented, with each prior step divided in half.

As to my preferred way of handling DSS scores over time, it remains the same as previously. Improvement or worsening for each patient was defined as a gain or loss of at least one step on the DSS. This should not happen unless at least one FS changed by an equivalent degree in the same direction. The plotting of cases at two intervals would then be most easily accomplished by a grid correlate of DSS scores at time 1 versus DSS scores at time 2. The numbers moving off the major diagonal of no change provide the numbers improving or worsening by one, two, three or more steps (figure). Then the proportions better-same-worse could be compared between two regimens if this were a therapeutic trial.

With the EDSS, a gain/loss of 0.5 steps will be defined as better/worse, but again, greater changes can be recorded. I cannot assert that each EDSS gain of 0.5 
should be accompanied by a change in FS of at least one grade, but I would be suspicious of the DSS change if this were not evident.

In other words, despite the Gaussian configuration of the DSS, I still prefer to treat it as an ordinal scale. For the FS, the only proper assessment is to consider each System individually, to plot "in" versus "out" as a grid correlate as with the DSS, and then to look at proportions changing in simile modo. Further, the FS scores are not additive, and each system can be compared only with itself. One obvious reason is that as Pyramidal worsens, Cerebellar will "improve," since patients cannot be ataxic if they cannot move. The lack of additivity in these systems was the underlying reason for the DSS. Also, I believe that mean FS scores are difficult to defend, even when speaking only to the individual systems. The distributions for most of them are clearly non-Gaussian (tables 3 through 6 ), and they also have differing configurations one versus another.

In the introduction, another impetus behind the paper was mentioned. The International Federation of Multiple Sclerosis Societies (IFMSS) is trying to establish a Uniform Minimum Record of Disability, which would be internationally acceptable as a way to characterize MS patients. ${ }^{10}$ Three separate scales were desired: one rating scheme to record the neurologic signs, one to record the physical disabilities or impairments, and one to record the societal impact of the disease. With differing labels, this follows the schema recommended by the World Health Organization to classify the consequences of disease according to "impairments" (neurologic abnormalities), "disabilities," and "handicaps."11

At a meeting in Stockholm ${ }^{12}$ it was thought that, for what by WHO was called (neurologic) "impairment," the rating scheme presented here-the DSS plus FS-was the most likely to meet with, if not universal acceptance, at least minimal opposition when compared with other proposals. The wide use of this method was documented. ${ }^{12}$ For the physical impairments or "disabilities" resulting from the disease, an Incapacity Scale was devised-a term chosen deliberately because it had not yet been appropriated by any other scheme. ${ }^{13}$ The societal impact (WHO: "handicaps") was assayed by what was then called a Socio-Economic Scale. ${ }^{14}$ Both the latter scales have been undergoing revisions, the economic one most drastically. IFMSS is continuing these efforts to establish and test a common tripartite scheme that would be suitable for all centers.

\section{Appendix A. Functional Systems.}

\section{Pyramidal Functions}

0 . Normal.

1. Abnormal signs without disability.

2. Minimal disability.

3. Mild or moderate paraparesis or hemiparesis; severe monoparesis.
4. Marked paraparesis or hemiparesis; moderate quadriparesis; or monoplegia.

5. Paraplegia, hemiplegia, or marked quadriparesis.

6. Quadriplegia.

V. Unknown.

\section{Cerebellar Functions}

0 . Normal.

1. Abnormal signs without disability.

2. Mild ataxia.

3. Moderate truncal or limb ataxia.

4. Severe ataxia, all limbs.

5. Unable to perform coordinated movements due to ataxia.

V. Unknown.

$X$. Is used throughout after each number when weakness (grade 3 or more on pyramidal) inter feres with testing.

\section{Brain Stem Functions}

0 . Normal.

1. Signs only.

2. Moderate nystagmus or other mild disability.

3. Severe nystagmus, marked extraocular weakness, or moderate disability of other cranial nerves.

4. Marked dysarthria or other marked disability.

5. Inability to swallow or speak.

V. Unknown.

\section{Sensory Functions (revised 1982)}

0 . Normal.

1. Vibration or figure-writing decrease only, in one or two limbs.

2. Mild decrease in touch or pain or position sense, and/or moderate decrease in vibration in one or two limbs; or vibratory (c/s figure writing) decrease alone in three or four limbs.

3. Moderate decrease in touch or pain or position sense, and/or essentially lost vibration in one or two limbs; or mild decrease in touch or pain and/or moderate decrease in all proprioceptive tests in three or four limbs.

4. Marked decrease in touch or pain or loss of proprioception, alone or combined, in one or two limbs; or moderate decrease in touch or pain and/or severe proprioceptive decrease in more than two limbs.

5. Loss (essentially) of sensation in one or two limbs; or moderate decrease in touch or pain and/or loss of proprioception for most of the body below the head.

6. Sensation essentially lost below the head.

V. Unknown.

\section{Bowel and Bladder Functions (revised 1982)}

0 . Normal.

1. Mild urinary hesitancy, urgency, or retention.

2. Moderate hesitancy, urgency, retention of bowel or bladder, or rare urinary incontinence. 
3. Frequent urinary incontinence.

4. In need of almost constant catheterization.

5. Loss of bladder function.

6. Loss of bowel and bladder function.

V. Unknown.

\section{Visual (or Optic) Functions}

\section{0 . Normal.}

1. Scotoma with visual acuity (corrected) better than 20/30.

2. Worse eye with scotoma with maximal visual acuity (corrected) of $20 / 30$ to $20 / 59$.

3 . Worse eye with large scotoma, or moderate decrease in fields, but with maximal visual acuity (corrected) of 20/60 to 20/99.

4. Worse eye with marked decrease of fields and maximal visual acuity (corrected) of $20 / 100$ to 20/200; grade 3 plus maximal acuity of better eye of $20 / 60$ or less.

5. Worse eye with maximal visual acuity (corrected) less than 20/200; grade 4 plus maximal acuity of better eye of $20 / 60$ or less.

6. Grade 5 plus maximal visual acuity of better eye of $20 / 60$ or less.

$\mathrm{V}$. Unknown.

$\mathrm{X}$. Is added to grades 0 to 6 for presence of temporal pallor.

\section{Cerebral (or Mental) Functions}

0 . Normal.

1. Mood alteration only (Does not affect DSS score).

2. Mild decrease in mentation.

3. Moderate decrease in mentation.

4. Marked decrease in mentation (chronic brain syndrome-moderate).

5. Dementia or chronic brain syndrome-severe or incompetent.

V. Unknown.

\section{Other Functions.}

0 . None.

1. Any other neurologic findings attributed to MS (specify).

V. Unknown.

\section{Appendix B. Expanded Disability Status Scale (EDSS)}

$0=$ Normal neurologic exam (all grade 0 in Functional Systems [FS]; Cerebral grade 1 acceptable).

$1.0=$ No disability, minimal signs in one FS (ie, grade 1 excluding Cerebral grade 1 ).

$1.5=$ No disability minimal signs in more than one FS (more than one grade 1 excluding Cerebral grade 1).
$2.0=$ Minimal disability in one FS (one FS grade 2, others 0 or 1 ).

$2.5=$ Minimal disability in two FS (two FS grade 2, others 0 or 1 ).

$3.0=$ Moderate disability in one FS (one FS grade 3, others 0 or 1 ), or mild disability in three or four FS (three/four FS grade 2, others 0 or 1) though fully ambulatory.

$3.5=$ Fully ambulatory but with moderate disability in one FS (one grade 3) and one or two FS grade 2; or two FS grade 3; or five FS grade 2 (others 0 or 1 ).

$4.0=$ Fully ambulatory without aid, self-sufficient, up and about some 12 hours a day despite relatively severe disability consisting of one FS grade 4 (others 0 or 1 ), or combinations of lesser grades exceeding limits of previous steps. Able to walk without aid or rest some 500 meters.

$4.5=$ Fully ambulatory without aid, up and about much of the day, able to work a full day, may otherwise have some limitation of full activity or require minimal assistance; characterized by relatively severe disability, usually consisting of one FS grade 4 (others 0 or 1) or combinations of lesser grades exceeding limits of previous steps. Able to walk without aid or rest for some 300 meters.

$5.0=$ Ambulatory without aid or rest for about 200 meters; disability severe enough to impair full daily activities (eg, to work full day without special provisions). (Usual FS equivalents are one grade 5 alone, others 0 or 1 ; or combinations of lesser grades usually exceeding specifications for step 4.0.)

$5.5=$ Ambulatory without aid or rest for about 100 meters; disability severe enough to preclude full daily activities. (Usual FS equivalents are one grade 5 alone, others 0 or 1 ; or combinations of lesser grades usually exceeding those for step 4.0.)

$6.0=$ Intermittent or unilateral constant assistance (cane, crutch, or brace) required to walk about 100 meters with or without resting. (Usual FS equivalents are combinations with more than two FS grade $3+$.)

$6.5=$ Constant bilateral assistance (canes, crutches, or braces) required to walk about 20 meters without resting. (Usual FS equivalents are combinations with more than two FS grade $3+$.) 
$7.0=$ Unable to walk beyond about 5 meters even with aid, essentially restricted to wheelchair; wheels self in standard wheelchair and transfers alone; up and about in $\mathrm{w} / \mathrm{c}$ some 12 hours a day. (Usual FS equivalents are combinations with more than one FS grade $4+$; very rarely, pyramidal grade 5 alone.)

$7.5=$ Unable to take more than a few steps; restricted to wheelchair; may need aid in transfer; wheels self but cannot carry on in standard wheelchair a full day; may require motorized wheelchair. (Usual FS equivalents are combinations with more than one FS grade $4+$.)

$8.0=$ Essentially restricted to bed or chair or perambulated in wheelchair, but may be out of bed itself much of the day; retains many self-care functions; generally has effective use of arms. (Usual FS equivalents are combinations, generally grade $4+$ in several systems.)

$8.5=$ Essentially restricted to bed much of the day; has some effective use of arm(s); retains some self-care functions. (Usual FS equivalents are combinations, generally $4+$ in several systems.)

$9.0=$ Helpless bed patient; can communicate and eat. (Usual FS equivalents are combinations, mostly grade $4+$.)

$9.5=$ Totally helpless bed patient; unable to communicate effectively or eat/swallow. (Usual FS equivalents are combinations, almost all grade $4+$.)

10. = Death due to MS.

\section{References}

1. Kurtzke JF. A new scale for evaluating disability in multiple sclerosis. Neurology (Minneap) 1955;5:580-3.

2. Kurtzke JF, Berlin L. The effects of isoniazid on patients with multiple sclerosis: preliminary report. AM Rev Tuberc 1954;70:577-92.

3. Veterans Administration Multiple Sclerosis Study Group. Isoniazid in treatment of multiple sclerosis. Report on Veterans Administration cooperative study. JAMA 1957;163:168-72.

4. Kurtzke JF, Berlin L. Isoniazid in treatment of multiple sclerosis. JAMA 1957;163:172-4.

5. Kurtzke JF. On the evaluation of disability in multiple sclerosis. Neurology (Minneap) 1961;11:686-94.

6. Kurtzke JF. Further notes on disability evaluation in multiple sclerosis, with scale modifications. Neurology (Minneap) $1965 ; 15: 654-61$.

7. Kurtzke JF, Beebe GW, Nagler B, Auth TL, Kurland LT, Nefzger MD. Studies on the natural history of multiple sclerosis. 6. Clinical and laboratory findings at first diagnosis. Acta Neurol Scand 1972;48:19-46.

8. Kurtzke JF. Neurologic impairment in multiple sclerosis and the Disability Status Scale. Acta Neurol Scand 1970;46:493-512.

9. Guthrie TC, Kurtzke JF, Berlin L. Acute respiratory failure in multiple sclerosis and its management. Ann Intern Med 1952;37:1197-203.

10. Slater RJ. International Symposium on MS services: introductory remarks. Functional disability ratings: medical, sacial and economic implications. Acta Neurol Scand (Suppl 87) $1981 ; 64: 3-4$

11. Wood PHN. Appreciating the consequences of disease: the international classification of impairments, disabilities, and handicaps. WHO Chron 1980;34:376-80.

12. Slater RJ, Fog T, Bergmann L. International Federation of Multiple Sclerosis Societies. A Festschrift for Professor Tore Broman. Symposium on multiple sclerosis services. Functional disability ratings: medical, social and economic implications. Stockholm, Sweden, August 31-September 1, 1980. Acta Neurol Scand (Suppl 87) 1981:64:1-138.

13. Kurtzke JF. A proposal for uniform minimal record of disability in multiple sclerosis. Acta Neurol Scand (Suppl 87) 1981;64:110-29.

14. Mellerup E, Fog T, Raun N, et al. The socio-economic scale. Acta Neurol Scand (Suppl 87) 1981;64:130-8. 


\title{
Neurology
}

\author{
Rating neurologic impairment in multiple sclerosis: An expanded disability \\ status scale (EDSS) \\ John F. Kurtzke \\ Neurology 1983;33;1444 \\ DOI 10.1212/WNL.33.11.1444
}

This information is current as of November 1,1983

\section{Updated Information \& \\ Services}

Citations

Permissions \& Licensing

Reprints including high resolution figures, can be found at: http://n.neurology.org/content/33/11/1444.full

This article has been cited by 100 HighWire-hosted articles:

http://n.neurology.org/content/33/11/1444.full\#\#otherartic les

Information about reproducing this article in parts (figures,tables) or in its entirety can be found online at: http://www.neurology.org/about/about_the_journal\#permi ssions

Information about ordering reprints can be found online: http://n.neurology.org/subscribers/advertise

Neurology ${ }^{\circledR}$ is the official journal of the American Academy of Neurology. Published continuously since 1951, it is now a weekly with 48 issues per year. Copyright (C) 1983 by the American Academy of Neurology. All rights reserved. Print ISSN: 0028-3878. Online ISSN: 1526-632X.

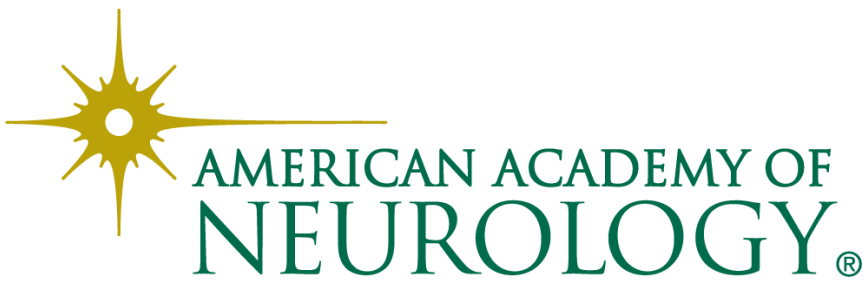

